\title{
A density functional theory study of magneto- electric Jones birefringence of noble gases, furan homologues, and mono-substituted benzenes
}

Tobias Fahleson, Patrick Norman, Sonia Coriani, Antonio Rizzo and Geert L J A Rikken

\author{
Linköping University Post Print
}

\section{Tweet}

N.B.: When citing this work, cite the original article.

Original Publication:

Tobias Fahleson, Patrick Norman, Sonia Coriani, Antonio Rizzo and Geert L J A Rikken, A density functional theory study of magneto-electric Jones birefringence of noble gases, furan homologues, and mono-substituted benzenes, 2013, Journal of Chemical Physics, (139), 19, 194311.

http://dx.doi.org/10.1063/1.4830412

Copyright: American Institute of Physics (AIP) http://www.aip.org/

Postprint available at: Linköping University Electronic Press

http://urn.kb.se/resolve?urn=urn:nbn:se:liu:diva-102851 


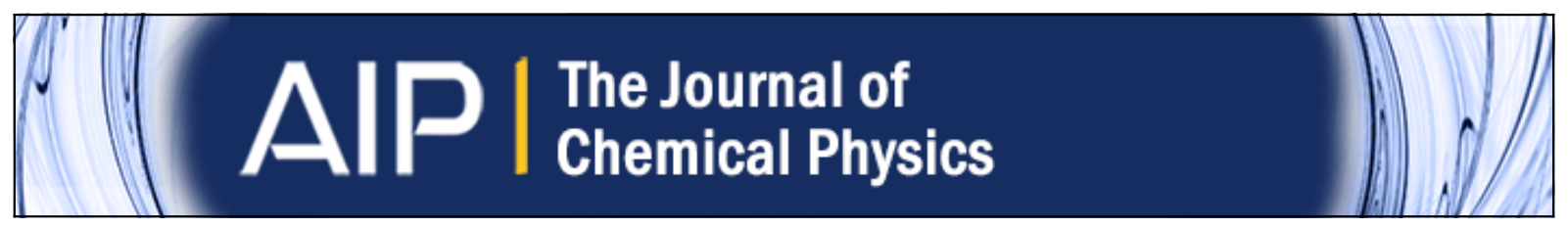

A density functional theory study of magneto-electric Jones birefringence of noble gases, furan homologues, and mono-substituted benzenes

Tobias Fahleson, Patrick Norman, Sonia Coriani, Antonio Rizzo, and Geert L. J. A. Rikken

Citation: The Journal of Chemical Physics 139, 194311 (2013); doi: 10.1063/1.4830412

View online: http://dx.doi.org/10.1063/1.4830412

View Table of Contents: http://scitation.aip.org/content/aip/journal/jcp/139/19?ver=pdfcov

Published by the AIP Publishing

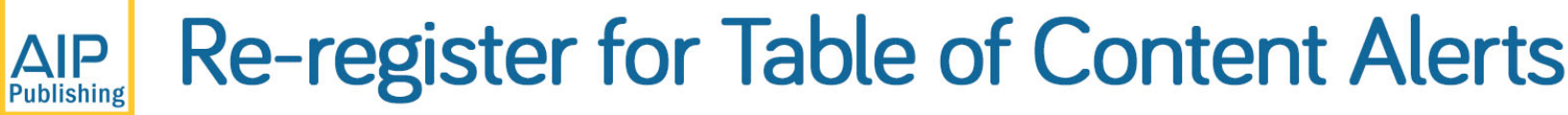

\section{Create a profile.}




\title{
A density functional theory study of magneto-electric Jones birefringence of noble gases, furan homologues, and mono-substituted benzenes
}

\author{
Tobias Fahleson, ${ }^{1}$ Patrick Norman, ${ }^{1, a)}$ Sonia Coriani, ${ }^{2, b)}$ Antonio Rizzo, ${ }^{3, c}$ \\ and Geert L. J. A. Rikken ${ }^{4, d)}$ \\ ${ }^{1}$ Department of Physics, Chemistry and Biology, Linköping University, SE-581 83 Linköping, Sweden \\ ${ }^{2}$ Dipartimento di Scienze Chimiche e Farmaceutiche, Università degli Studi di Trieste, I-34127 Trieste, Italy \\ ${ }^{3}$ CNR - Consiglio Nazionale delle Ricerche, Istituto per i Processi Chimico Fisici (IPCF-CNR), UOS di Pisa, \\ I-56124 Pisa, Italy \\ ${ }^{4}$ Laboratoire National des Champs Magnétiques Intenses, UPR3228, CNRS/INSA/UJF/UPS, Toulouse and \\ Grenoble, France
}

(Received 25 September 2013; accepted 1 November 2013; published online 20 November 2013)

\begin{abstract}
We report on the results of a systematic ab initio study of the Jones birefringence of noble gases, of furan homologues, and of monosubstituted benzenes, in the gas phase, with the aim of analyzing the behavior and the trends within a list of systems of varying size and complexity, and of identifying candidates for a combined experimental/theoretical study of the effect. We resort here to analytic linear and nonlinear response functions in the framework of time-dependent density functional theory. A correlation is made between the observable (the Jones constant) and the atomic radius for noble gases, or the permanent electric dipole and a structure/chemical reactivity descriptor as the para Hammett constant for substituted benzenes. ( 2013 AIP Publishing LLC. [http://dx.doi.org/10.1063/1.4830412]
\end{abstract}

\section{INTRODUCTION}

The interaction of light in its various polarization states with matter in the presence of external fields can give rise to a large number of different phenomena, some of which not yet fully explored. The response of matter to the combination of static and dynamic electromagnetic fields depends on the angular frequency and intensity of the radiation, the strength of the applied external fields, the conditions of temperature and pressure, as well as the structural properties of its constituents. The field of research is vast, and of importance not only for its implications in the understanding of the foundations of the interaction of light and matter, but also for its technological spillovers. The need for tracking down often tiny signatures of novel or yet undetected phenomena triggers new developments in the design of ever more powerful detection techniques, ever more sophisticated optical arrangements, ever stronger field sources, and new signal analysis and characterization routines. From the theoretical point of view, electromagnetic fields interacting with atoms and molecules probe their electronic structure, provoking responses bearing unique signatures of the nuclear arrangement and of the distribution of the electron density in the constituents of matter. Their study puts to a test the techniques and algorithms developed by theoreticians in the last decades to compute frequency dependent, often high-order, response properties, and, with the growing interest in systems of large size, it has also forced the development of approximations which could make

\footnotetext{
a)panor@ifm.liu.se

b) coriani@units.it

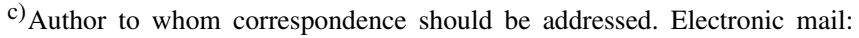
rizzo@ipcf.cnr.it

d) geert.rikken@lncmi.cnrs.fr
}

calculations affordable when the number of nuclei and electrons increases.

Birefringences (the anisotropies induced in the real part of the complex refractive index with respect to two directions of polarization-either linear or circular in the case of linear or circular birefringence - or propagation in spacefor axial birefringences) and dichroisms (the corresponding anisotropies observed in the imaginary part of the refractive index) are two general examples of optical phenomena we are referring to in this discussion. ${ }^{1-3}$ In some well known cases, there is no need for external fields for these phenomena to occur, since they arise from the intrinsic properties of matter, and in particular from its symmetry. Natural optical rotation $(\mathrm{OR})^{4,5}$ or circular dichroism (CD), ${ }^{6,7}$ discovered and rationalized theoretically well before the current times, are common examples of a circular birefringence and dichroism, respectively. These phenomena, occurring in assemblies of chiral molecules, can be rationalized theoretically by invoking, in a perturbative framework for the interaction of light and matter, the effect of linear mixed electric and magnetic frequency dependent polarizabilities. The application of additional perturbations, as it happens when external and not necessarily uniform electric and magnetic fields are introduced in the experimental design, yields further complications to the picture, implying the combination of first-order, linear and nonlinear properties, bearing the signature of the various multipoles excited by the combined effect of light and fields. ${ }^{1,3}$ Examples which have been studied in some depth in our groups are Kerr, ${ }^{8,9}$ Optical Kerr, ${ }^{10-12}$ Cotton-Mouton, ${ }^{13-20}$ Buckingham, ${ }^{21-27}$ magnetoelectric and Jones ${ }^{28-34}$ linear birefringences; Faraday ${ }^{4,35-37}$ and optical Faraday ${ }^{38}$ circular birefringences; magnetochiral axial birefringence; $;^{39,40}$ or magnetic circular ${ }^{41-49}$ and magnetochiral ${ }^{39,50-53}$ dichroism. 
In this paper, we concentrate on Jones and magnetoelectric linear birefringences, ${ }^{28-34}$ observable when linearly polarized light impinges on a uniaxial crystal or on homogeneous fluid samples subjected to both external electric and magnetic fields, aligned parallel (Jones) or perpendicular (magnetoelectric) to each other, and perpendicular to the direction of propagation of light. These two birefringences, differing in the experimental arrangement, yield equivalent expressions for the observable, ${ }^{29,31}$ and are therefore indistinguishable from the theoretical and computational point of view. From now on we will refer uniquely to Jones birefringence in our discussion. The characteristics of Jones birefringence are a bilinearity in the electric and magnetic field strengths and the fact that the optical axes defining the observable (the two directions with respect to which the linear birefringence is determined) are at $\pm 45^{\circ}$ with respect to those of the standard birefringence to which it superimposes, i.e., Kerr (electric) ${ }^{8,9}$ and Cotton-Mouton (magnetic) ${ }^{13-20}$ double refractions. Jones birefringence owes its denomination to R. C. Jones ${ }^{28}$ who first predicted its existence in his seminal contribution dated 1948. A complete theoretical rationalization is due to Graham and Raab, ${ }^{29}$ and it dates 1983. The same authors have shown the equivalence in theory of Jones and the magnetoelectric "variant" one year later. ${ }^{31}$ The latter had been predicted by Pockels $\mathrm{s}^{30}$ and Kielich ${ }^{32}$ and discussed by Baranova and co-workers. ${ }^{33}$ Ross, Sherborne, and Stedman ${ }^{34}$ contributed a few years later to the further comprehension of the symmetry rules governing the effect. Rizzo and Coriani ${ }^{54}$ in 2003 translated Graham and Raab expressions into a computationally affordable protocol, enabling the estimate of the observable in isotropic assemblies of noninteracting diamagnetic molecules. They resorted to modern analytic linear and nonlinear response theory, and since its first appearance their approach has been applied to a selection of systems of increasing complexity, and extended from the original independent particle (Hartree-Fock) and highly correlated (Coupled Cluster, CC) structural model to the Time-Dependent Density Functional Theory (TDDFT) ${ }^{55,56}$ realm. The latter permits the study of relatively extended systems with an often well satisfying account of the effect of electron correlation, ${ }^{57,58}$ see also Ref. 2. In the meantime, aspects as the dependence on the accurate (beyond $\mathrm{CC}$ singles and doubles, $\mathrm{CCSD}^{59}$ ) treatment of electron correlation, ${ }^{60}$ the effect of molecular vibrations (in diatomics), ${ }^{61}$ that of the environment, ${ }^{62}$ or the dependence of the results of calculations performed in finite one-electron basis sets on the choice of the magnetic gauge origin ${ }^{63}$ have been also studied. The theory of magnetoelectric Jones birefringence and dichroism was redrafted by Mironova and coworkers ${ }^{64}$ a few years ago. Very recently, there has also been some controversy on the existence of such an effect. ${ }^{65}$

Experimental verification of the predictions of Jones dates the beginning of this century, and is due to one of the present authors and his group. ${ }^{66-70}$ Rikken and Roth ${ }^{66}$ have measured Jones birefringence in liquid samples, obtaining upper limits to the expected birefringence for some of the systems analyzed in the study. The results of their measurement have been compared with computational estimates in Ref. 62.

In this paper, we report on the results of a systematic $a b$ initio investigation of the Jones birefringence of noble gases, furan homologues, and monosubstituted benzenes in the gas phase. The aim being to analyze the behavior and the trends within a list of systems of varying size and complexity, we resort here to TDDFT ${ }^{55,56}$ linear $^{71}$ and nonlinear ${ }^{71,72}$ response theory. TDDFT has recently proven in various instances to be able to reproduce quite satisfactorily spectroscopic linear and nonlinear properties of molecules of reasonable size and complexity, see, for example, Ref. 73, and we have employed it for calculations of Jones birefringence in the past. ${ }^{2,57,58,62} \mathrm{~A}$ correlation is made between the observable (the Jones constant, see Sec. II), and the atomic radius (for noble gases) or the permanent electric dipole moment or such a qualitative/quantitative structure chemical reactivity descriptor as the para Hammett constant, ${ }^{74,75}$ for substituted benzenes.

In Sec. II, definitions and theoretical expressions for the observable are given. Section III briefly recapitulates the computational details. The results are presented and discussed in Sec. IV and some brief conclusions are given at the end.

\section{METHODOLOGY}

The expression for the anisotropy of the refractive index in the case of Jones birefringence takes the following form: ${ }^{29,54,62}$

$$
\begin{aligned}
\Delta n= & n_{-45^{\circ}}-n_{+45^{\circ}}=\frac{N E_{x} B_{x}}{30 \epsilon_{0} c_{0}} \\
& \times\left\{\mathcal{G}_{\text {para }}^{(3)}+\mathcal{G}_{\text {dia }}^{(3)}+\mathcal{A}^{(3)}+\frac{1}{k_{\mathrm{B}} T}\left[\mathcal{G}_{\mathrm{para}}^{(2)}+\mathcal{G}_{\text {dia }}^{(2)}+\mathcal{A}^{\prime(2)}\right]\right\},
\end{aligned}
$$

where

$$
\begin{gathered}
\mathcal{G}_{\text {para }}^{(3)}=3 G_{\alpha \beta \alpha \beta}^{\mathrm{para}}+3 G_{\alpha \beta \beta \alpha}^{\mathrm{para}}-2 G_{\alpha \alpha \beta \beta}^{\mathrm{para}}, \\
\mathcal{G}_{\text {dia }}^{(3)}=3 G_{\alpha \beta \alpha \beta}^{\mathrm{dia}}+3 G_{\alpha \beta \beta \alpha}^{\mathrm{dia}}-2 G_{\alpha \alpha \beta \beta}^{\mathrm{dia}}, \\
\mathcal{A}^{\prime(3)}=-\frac{\omega}{2} \epsilon_{\alpha \beta \gamma}\left(a_{\alpha \beta \delta \delta \gamma}^{\prime}+a_{\alpha \beta \delta \gamma \delta}^{\prime}\right), \\
\mathcal{G}_{\text {para }}^{(2)}=\mu_{\alpha}\left(3 G_{\alpha \beta \beta}^{\mathrm{para}}+3 G_{\beta \alpha \beta}^{\mathrm{para}}-2 G_{\beta \beta \alpha}^{\mathrm{para}}\right), \\
\mathcal{G}_{\text {dia }}^{(2)}=\mu_{\alpha}\left(3 G_{\alpha \beta \beta}^{\mathrm{dia}}+3 G_{\beta \alpha \beta}^{\mathrm{dia}}-2 G_{\beta \beta \alpha}^{\mathrm{dia}}\right), \\
\mathcal{A}^{(2)}=-\frac{\omega}{2} \epsilon_{\alpha \beta \gamma}\left(\mu_{\gamma} a_{\alpha \beta \delta \delta}^{\prime}+\mu_{\delta} a_{\alpha \beta \delta \gamma}^{\prime}\right) .
\end{gathered}
$$

$N$ is the number density, $\epsilon_{0}$ is the permittivity of vacuum, $c_{0}$ is the speed of light in vacuo, $E_{x}$ and $B_{x}$ are the $x$-components of the external electric and magnetic fields. It is thus assumed that the radiation propagates in the $z$-direction. Other definitions include $\omega$, the circular frequency of the incoming radiation; $k_{\mathrm{B}}$, the Boltzmann constant; $T$, the temperature; $\mu_{\alpha}$, the permanent electric dipole in the $\alpha$-direction; and $\epsilon_{\alpha \beta \gamma}$, the Levi-Civita alternating tensor. Implicit summation over repeated indices applies. Atomic units are used here and, unless specified otherwise, also elsewhere in the present work. 
The individual elements included in the tensor contractions of Eqs. (2)-(7) are defined as

$$
\begin{aligned}
G_{\alpha \beta \gamma \delta}^{\mathrm{para}}=G_{\alpha \beta \gamma \delta}^{\mathrm{para}}(-\omega ; \omega, 0,0) & =-\left\langle\left\langle\hat{\mu}_{\alpha} ; \hat{m}_{\beta}, \hat{m}_{\gamma}, \hat{\mu}_{\delta}\right\rangle\right\rangle_{\omega, 0,0}, \\
G_{\alpha \beta \gamma \delta}^{\mathrm{dia}}=G_{\alpha \beta \gamma \delta}^{\mathrm{dia}}(-\omega ; \omega, 0) & =\left\langle\left\langle\hat{\mu}_{\alpha} ; \hat{\xi}_{\beta \gamma}^{\mathrm{dia}}, \hat{\mu}_{\delta}\right\rangle\right\rangle_{\omega, 0}, \\
a_{\alpha \beta \gamma \delta \epsilon}^{\prime}=a_{\alpha \beta \gamma \delta \epsilon}^{\prime}(-\omega ; \omega, 0,0) & =-i\left\langle\left\langle\hat{\mu}_{\alpha} ; \hat{q}_{\beta \gamma}, \hat{m}_{\delta}, \hat{\mu}_{\epsilon}\right\rangle\right\rangle_{\omega, 0,0}, \\
G_{\alpha \beta \gamma}^{\mathrm{para}}=G_{\alpha \beta \gamma}^{\mathrm{para}}(-\omega ; \omega, 0) & =\left\langle\left\langle\hat{\mu}_{\alpha} ; \hat{m}_{\beta}, \hat{m}_{\gamma}\right\rangle\right\rangle_{\omega, 0}, \\
G_{\alpha \beta \gamma}^{\mathrm{dia}}=G_{\alpha \beta \gamma}^{\mathrm{dia}}(-\omega ; \omega) & =-\left\langle\left\langle\hat{\mu}_{\alpha} ; \hat{\xi}_{\beta \gamma}^{\mathrm{dia}}\right\rangle\right\rangle_{\omega}, \\
a_{\alpha \beta \gamma \delta}^{\prime}=a_{\alpha \beta \gamma \delta}^{\prime}(-\omega ; \omega, 0) & =i\left\langle\left\langle\hat{\mu}_{\alpha} ; \hat{q}_{\beta \gamma}, \hat{m}_{\delta}\right\rangle\right\rangle_{\omega, 0},
\end{aligned}
$$

showing how they are identified as response functions holding for real electronic wave functions. The operators in the response functions above are

$$
\begin{gathered}
\text { electric dipole: } \hat{\mu}_{\alpha}=-\sum_{i} r_{i \alpha}, \\
\text { magnetic dipole: } \hat{m}_{\alpha}=-\frac{1}{2} \sum_{i}\left(r_{i} \times p_{i}\right)_{\alpha},
\end{gathered}
$$

traced electric quadrupole: $\hat{q}_{\alpha \beta}=-\sum_{i} r_{i \alpha} r_{i \beta}$,

diamagnetic susceptibility : $\hat{\xi}_{\beta \gamma}^{\mathrm{dia}}=\frac{1}{4} \sum_{i}\left(r_{i \beta} r_{i \gamma}-r_{i \delta} r_{i \delta} \delta_{\beta \gamma}\right)$.

Here, $i$ represents the $i$ th electron and $\alpha, \beta, \gamma$, and $\delta$ any of the cartesian coordinates $x, y$, and $z . r_{i \alpha}$ and $p_{i \alpha}$ are the components of the position and linear momentum operators, respectively.

Equation (1) may be rewritten in a more compact form,

$$
\Delta n=n_{-45^{\circ}}-n_{+45^{\circ}}=\frac{N E_{x} B_{x}}{30 \epsilon_{0} c_{0}} \times\left\{\mathrm{J}_{0}+\frac{\mathrm{J}_{T}}{T}\right\},
$$

where, rather trivially

$$
\begin{gathered}
\mathrm{J}_{0}=\mathcal{G}_{\text {para }}^{(3)}+\mathcal{G}_{\text {dia }}^{(3)}+\mathcal{A}^{\prime(3)}, \\
\mathrm{J}_{T}=\frac{1}{k_{\mathrm{B}}}\left[\mathcal{G}_{\text {para }}^{(2)}+\mathcal{G}_{\text {dia }}^{(2)}+\mathcal{A}^{(2)}\right] .
\end{gathered}
$$

For an ideal gas at pressure $P$, Eq. (9) becomes

$$
\Delta n=\frac{P E_{x} B_{x}}{30 \epsilon_{0} c_{0} k_{\mathrm{B}} T} \times\left\{\mathrm{J}_{0}+\frac{\mathrm{J}_{T}}{T}\right\} .
$$

The Jones constant $k_{\mathrm{J}}$ may be defined as

$$
\begin{aligned}
k_{\mathrm{J}} & =\frac{P}{30 \epsilon_{0} c_{0} \lambda k_{\mathrm{B}} T} \times\left\{\mathrm{J}_{0}+\frac{\mathrm{J}_{T}}{T}\right\} \\
& \approx 2.75028 \times 10^{-14} \times \frac{P[\mathrm{~atm}]}{\lambda[\mathrm{nm}] \times T[\mathrm{~K}]} \times\left\{\mathrm{J}_{0}+\frac{\mathrm{J}_{T}}{T}\right\} \text { [a.u.] }
\end{aligned}
$$

Above, $k_{\mathrm{J}}$ is obtained in SI units of $\mathrm{V}^{-1} \mathrm{~T}^{-1}$ with the choice of units specified in square brackets in Eq. (13). With the newly defined constant, Eq. (12) may be recast in a further reduced form as

$$
\Delta n=n_{-45^{\circ}}-n_{+45^{\circ}}=k_{\mathrm{J}} \lambda E_{x} B_{x} .
$$

\section{COMPUTATIONAL DETAILS}

All calculations were carried out for molecular structures optimized at the level of Kohn-Sham density functional theory (DFT) in conjunction with the hybrid B3LYP ${ }^{76-78}$ exchange-correlation functional and Dunning's triple- $\zeta$ $(\mathrm{cc}-\mathrm{pVTZ})^{79}$ basis set. For the property calculations, we have made use of the Coulomb-attenuated method B3LYP (CAM-B3LYP) exchange correlation functional ${ }^{80-82}$ to properly account for charge-transfer excitations-an issue that becomes important for the mono-substituted benzenes. We have adopted Dunning's t-aug-cc-pVQZ and t-aug-pVTZ basis sets ${ }^{79,83,84}$ for property calculations on noble gas atoms and polyatomic molecules, respectively. For xenon, the t-augcc-pVQZ-pp basis set and effective small-core relativistic potential were employed. ${ }^{85}$ Molecular structure optimizations and property calculations have been carried out with use of the Gaussian ${ }^{86}$ and DALTON ${ }^{87}$ programs, respectively.

\section{RESULTS AND DISCUSSION}

Table I summarizes the quantities relevant for the analysis of the Jones birefringence of the systems chosen for this study, i.e., the noble gases $\mathrm{He}$ to $\mathrm{Xe}$, and a set of molecules including furan and its homologues thiophene and selenophene, benzene and eleven mono-substituted benzenes. In the table, the Jones constant-in particular the product of $k_{\mathrm{J}}$ and the wavelength $\lambda$, cf. Eq. (13)-is given together with the $\mathrm{J}_{0}$ and $\mathbf{J}_{T} / T$ contributions, defined in Eqs. (9)-(11), with the isotropic dynamic polarizability $\alpha_{\mathrm{av}}(-\omega ; \omega)$ and the permanent electric dipole moment $\mu$. The results were obtained at TDDFT level by employing the standard form of the CAM-B3LYP functional, assuming a wavelength of $632.8 \mathrm{~nm}$, and, where relevant, a pressure of $1 \mathrm{~atm}$, and a temperature of $294.15 \mathrm{~K}$. Table II reports the contributions, Eqs. (2)-(7), to $\mathrm{J}_{0}$ and $\mathrm{J}_{T} / T$. The geometry of the molecules studied in this work, with each individual dipole vector aligned with the $z$-axis, is shown in Figure 1. In Table I, the ratio $k_{\mathrm{J}} \lambda / \alpha$, relative to Helium, and employed here as the Figure of Merit (FOM) is reported. In earlier work, ${ }^{66}$ the ratio

$$
\eta=\frac{k_{\mathrm{J}}}{\sqrt{k_{\mathrm{CM}} k_{\mathrm{K}}}}
$$

has been used as an index of the measurability of Jones birefringence. Above $k_{\mathrm{CM}}$ and $k_{\mathrm{K}}$ are the Cotton-Mouton and Kerr constants, respectively, defined through the relationships

$$
\begin{gathered}
\Delta n_{\mathrm{CM}}=k_{\mathrm{CM}} \lambda B^{2}, \\
\Delta n_{\mathrm{K}}=k_{\mathrm{K}} \lambda E^{2} .
\end{gathered}
$$

The quantity $\eta$ therefore gives a measure of the size of the Jones/magnetoelectric birefringence, when subject to both electric $(E)$ and magnetic $(B)$ fields simultaneously, compared to the supposedly stronger effects which are observed in presence of either $E$ (for Kerr) or $B$ (for CME) alone. For such a quantity, early, very approximate estimates by Graham and $\mathrm{Raab}^{29}$ and Ross and co-workers ${ }^{34}$ predict values of the order of twice the fine structure constant $\left(\alpha_{\mathrm{fs}} \approx 0.01459\right)$. Our calculations on noble gases, cf. Refs. 2, 54, and 60, 
TABLE I. Isotropic dynamic polarizability $\alpha_{\mathrm{av}}(-\omega ; \omega)$ [a.u.], dipole moment $\mu$ [a.u.], and Jones constant times the photon wavelength $k_{\mathrm{J}} \lambda\left[10^{-20} \mathrm{~m} \mathrm{~V}^{-1} \mathrm{~T}^{-1}\right.$ ] for noble gases, furan homologues, and mono-substituted benzenes. The quantities $\mathrm{J}_{0}$ and $\mathrm{J}_{T} / T$ constitute temperature independent and dependent parts of $k_{\mathrm{J}}$, respectively, and are both given in atomic units [a.u.]. Calculations refer to a wavelength of $632.8 \mathrm{~nm}$, pressure of 1 atm, and temperature of $294.15 \mathrm{~K}$, and are carried out at the CAM-B3LYP level of theory.

\begin{tabular}{|c|c|c|c|c|c|c|}
\hline & $\alpha_{\mathrm{av}}$ & $\mu$ & $\mathrm{J}_{0}$ & $\mathbf{J}_{T} / T$ & $k_{\mathrm{J}} \lambda$ & $\mathrm{FOM}^{\mathrm{a}}$ \\
\hline $\mathrm{He}$ & 1.5 & 0 & -79.9 & 0 & -0.00075 & 1 \\
\hline $\mathrm{Ne}$ & 2.9 & 0 & -177.1 & 0 & -0.0017 & 1.2 \\
\hline $\mathrm{Ar}$ & 11.7 & 0 & -1277.6 & 0 & -0.012 & 2.1 \\
\hline $\mathrm{Kr}$ & 17.8 & 0 & -2558.8 & 0 & -0.024 & 2.8 \\
\hline $\mathrm{Xe}$ & 28.6 & 0 & -5654.5 & 0 & -0.053 & 3.6 \\
\hline $\mathrm{C}_{4} \mathrm{H}_{4} \mathrm{O}$ & 49.8 & 0.27 & 1773.6 & 11389.7 & 0.12 & 5.1 \\
\hline $\mathrm{C}_{4} \mathrm{H}_{4} \mathrm{~S}$ & 65.9 & 0.22 & 1247.6 & -772.0 & 0.0044 & 0.1 \\
\hline $\mathrm{C}_{4} \mathrm{H}_{4} \mathrm{Se}$ & 73.3 & 0.18 & 770.9 & -6997.3 & -0.058 & 1.6 \\
\hline $\mathrm{C}_{6} \mathrm{H}_{5} \mathrm{NH}_{2}$ & 83.7 & -0.6 & 13730.9 & 43203.0 & 0.53 & 13.1 \\
\hline $\mathrm{C}_{6} \mathrm{H}_{5} \mathrm{OH}$ & 76.9 & -0.51 & 7451.8 & -5243.3 & 0.021 & 0.6 \\
\hline $\mathrm{C}_{6} \mathrm{H}_{5} \mathrm{CH}_{3}$ & 84.7 & -0.16 & 6855.8 & 1213.8 & 0.075 & 1.8 \\
\hline $\mathrm{C}_{6} \mathrm{H}_{6}$ & 71.0 & 0 & 4335.4 & 0 & 0.041 & 1.2 \\
\hline $\mathrm{C}_{6} \mathrm{H}_{5} \mathrm{~F}$ & 71.0 & 0.63 & 4050.0 & -3152.0 & 0.0084 & 0.2 \\
\hline $\mathrm{C}_{6} \mathrm{H}_{5} \mathrm{Cl}$ & 86.4 & 0.72 & 7130.1 & -68234.3 & -0.57 & 13.6 \\
\hline $\mathrm{C}_{6} \mathrm{H}_{5} \mathrm{Br}$ & 94.8 & 0.74 & 7913.9 & -128306.8 & -1.13 & 24.4 \\
\hline $\mathrm{C}_{6} \mathrm{H}_{5} \mathrm{COH}$ & 89.1 & 1.37 & 8490.9 & -134679.9 & -1.18 & 27.2 \\
\hline $\mathrm{C}_{6} \mathrm{H}_{5} \mathrm{CN}$ & 89.1 & 1.87 & 7171.2 & -182532.7 & -1.64 & 37.9 \\
\hline $\mathrm{C}_{6} \mathrm{H}_{5} \mathrm{NO}_{2}$ & 90.0 & 1.86 & 7811.5 & 9699.6 & 0.16 & 3.7 \\
\hline $\mathrm{C}_{6} \mathrm{H}_{5} \mathrm{NO}$ & 87.7 & 1.48 & 56733.9 & 2274271.2 & 21.79 & 511.4 \\
\hline $\mathrm{C}_{6} \mathrm{H}_{5} \mathrm{CHC}(\mathrm{CN})_{2}$ & 149.2 & 2.69 & 23675.3 & -196979.2 & -1.62 & 22.3 \\
\hline
\end{tabular}

${ }^{a}$ The figure of merit (FOM) is defined as $\left|k_{\mathrm{J}} \lambda / \alpha\right|$ and reported relative He.

yield $\eta$ of the order of the fine structure constant (vide infra for a note on these results). For molecules, in line with experiment, ${ }^{66}$ the values of $\eta$ are much smaller than $\alpha_{\mathrm{fs}}$. Here, we resort to the FOM, as defined above, and we look for systems with high values of the FOM. In experiment, it is usually the relative change in the measured quantity, induced by the effect studied, that determines the detection limit.

\section{A. Noble gases}

The Jones birefringence of the noble gases $\mathrm{He}$ to $\mathrm{Kr}$ was studied by means of $a b$ initio theory in Ref. 54, where a coupled cluster model was employed for the calculation of the property, and the dependence on the extension and quality of the basis set was analyzed in detail. The results were further analyzed in Refs. 2 and 60. Rather unfortunately, a couple

TABLE II. The contributions to Jones birefringence, in a.u. Powers of ten are given in parentheses.

\begin{tabular}{|c|c|c|c|c|c|c|}
\hline & $\mathcal{A}^{\prime(3)}$ & $\mathcal{G}_{\text {para }}^{(3)}$ & $\mathcal{G}_{\text {dia }}^{(3)}$ & $\mathcal{A}^{\prime(2)}$ & $\mathcal{G}_{\text {para }}^{(2)}$ & $\mathcal{G}_{\text {dia }}^{(2)}$ \\
\hline $\mathrm{He}$ & $-1.0481(+00)$ & $1.5571(+01)$ & $-9.4410(+01)$ & & & \\
\hline $\mathrm{Ne}$ & $-3.0928(+00)$ & $2.9091(+01)$ & $-2.0307(+02)$ & & & \\
\hline $\mathrm{Ar}$ & $-4.8599(+01)$ & $2.5386(+02)$ & $-1.4828(+03)$ & & & \\
\hline $\mathrm{Kr}$ & $-1.2868(+02)$ & $5.1468(+02)$ & $-2.9448(+03)$ & & & \\
\hline $\mathrm{Xe}$ & $-3.9815(+02)$ & $1.1709(+03)$ & $-6.4272(+03)$ & & & \\
\hline $\mathrm{C}_{4} \mathrm{H}_{4} \mathrm{O}$ & $-3.1546(+02)$ & $1.1805(+04)$ & $-9.7156(+03)$ & $8.3160(-01)$ & $-5.0059(+00)$ & $1.4784(+01)$ \\
\hline $\mathrm{C}_{4} \mathrm{H}_{4} \mathrm{~S}$ & $-6.0594(+02)$ & $1.5464(+04)$ & $-1.3611(+04)$ & $3.9506(-01)$ & $-1.5969(+01)$ & $1.4855(+01)$ \\
\hline $\mathrm{C}_{4} \mathrm{H}_{4} \mathrm{Se}$ & $-8.1042(+02)$ & $1.7798(+04)$ & $-1.6216(+04)$ & $8.2357(-01)$ & $-4.2799(+01)$ & $3.5458(+01)$ \\
\hline $\mathrm{C}_{6} \mathrm{H}_{5} \mathrm{NH}_{2}$ & $-4.2662(+02)$ & $3.0927(+04)$ & $-1.6770(+04)$ & $-2.6532(+00)$ & $6.0975(+01)$ & $-1.8078(+01)$ \\
\hline $\mathrm{C}_{6} \mathrm{H}_{5} \mathrm{OH}$ & $-6.0618(+02)$ & $2.2995(+04)$ & $-1.4937(+04)$ & $9.7278(-01)$ & $-3.0533(+01)$ & $2.4676(+01)$ \\
\hline $\mathrm{C}_{6} \mathrm{H}_{5} \mathrm{CH}_{3}$ & $-6.0823(+02)$ & $2.3684(+04)$ & $-1.6220(+04)$ & $-6.5974(-01)$ & $2.7434(+00)$ & $-9.5295(-01)$ \\
\hline $\mathrm{C}_{6} \mathrm{H}_{6}$ & $-6.1055(+02)$ & $1.8602(+04)$ & $-1.3656(+04)$ & & & \\
\hline $\mathrm{C}_{6} \mathrm{H}_{5} \mathrm{~F}$ & $-4.9955(+02)$ & $1.6616(+04)$ & $-1.2067(+04)$ & $4.3384(+00)$ & $-1.0583(+02)$ & $9.8551(+01)$ \\
\hline $\mathrm{C}_{6} \mathrm{H}_{5} \mathrm{Cl}$ & $-6.7098(+02)$ & $2.3752(+04)$ & $-1.5951(+04)$ & $5.2600(+00)$ & $-1.8169(+02)$ & $1.1287(+02)$ \\
\hline $\mathrm{C}_{6} \mathrm{H}_{5} \mathrm{Br}$ & $-9.5886(+02)$ & $2.8219(+04)$ & $-1.9346(+04)$ & $9.3722(+00)$ & $-4.4480(+02)$ & $3.1591(+02)$ \\
\hline $\mathrm{C}_{6} \mathrm{H}_{5} \mathrm{CHO}$ & $-5.9849(+02)$ & $2.4585(+04)$ & $-1.5496(+04)$ & $1.5584(+01)$ & $-4.5826(+02)$ & $3.1722(+02)$ \\
\hline $\mathrm{C}_{6} \mathrm{H}_{5} \mathrm{CN}$ & $-6.2176(+02)$ & $2.3115(+04)$ & $-1.5322(+04)$ & $9.6514(+00)$ & $-1.1033(+02)$ & $-6.9351(+01)$ \\
\hline $\mathrm{C}_{6} \mathrm{H}_{5} \mathrm{NO}_{2}$ & $-5.0366(+02)$ & $2.3087(+04)$ & $-1.4771(+04)$ & $2.3168(+01)$ & $-4.9776(+02)$ & $4.8363(+02)$ \\
\hline $\mathrm{C}_{6} \mathrm{H}_{5} \mathrm{NO}$ & $-1.1154(+03)$ & $7.3058(+04)$ & $-1.5209(+04)$ & $1.1333(+00)$ & $1.6564(+03)$ & $4.6099(+02)$ \\
\hline $\mathrm{C}_{6} \mathrm{H}_{5} \mathrm{CHC}(\mathrm{CN})_{2}$ & $-1.4111(+03)$ & $4.7895(+04)$ & $-2.2809(+04)$ & $3.6289(+01)$ & $-2.7884(+02)$ & $5.9058(+01)$ \\
\hline
\end{tabular}



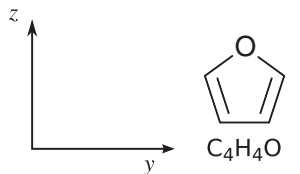

$\mathrm{C}_{4} \mathrm{H}_{4} \mathrm{O} \quad \mathrm{C}_{4} \mathrm{H}_{4} \mathrm{~S}$

Furan Thiophene Selenophene

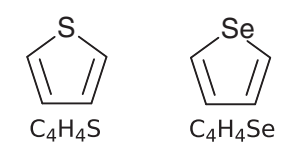

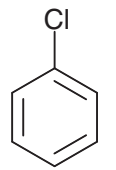

$\mathrm{C}_{6} \mathrm{H}_{5} \mathrm{Cl}$

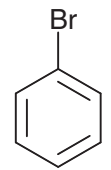

$\mathrm{C}_{6} \mathrm{H}_{5} \mathrm{Br}$

$\mathrm{C}_{6} \mathrm{H}_{5} \mathrm{~F}$

Fluorobenzene

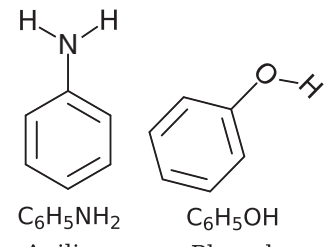

Phenol

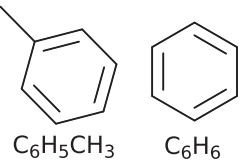

Toluene Benzene

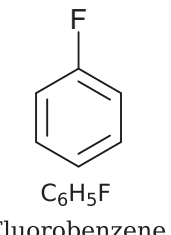

Chlorobenzene

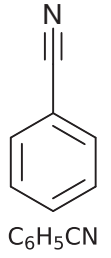

$\mathrm{C}_{6} \mathrm{H}_{5} \mathrm{COH}$

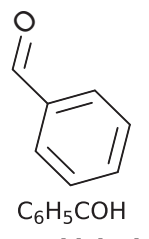

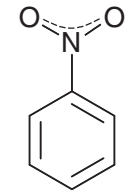

$\mathrm{C}_{6} \mathrm{H}_{5} \mathrm{NO}_{2}$

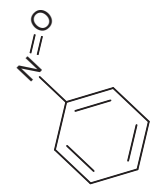

$\mathrm{C}_{6} \mathrm{H}_{5} \mathrm{NO}$

Nitrosobenzene

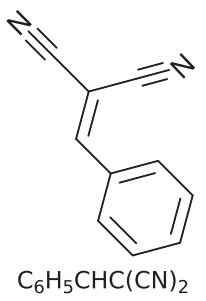

Benzal

FIG. 1. Geometry of molecules studied in this work, with each individual dipole vector aligned with the z-axis; its sign and value may be found in Table I. The tilt of the dipole relative to the substituent has been exaggerated for clarity, and it should be noted that the $2 \mathrm{D}$-nature of this figure does not convey that the dipole of aniline is not confined to the plane of the figure.

of errors slipped through in Sec. II A 1 of Ref. 54, cf. also Ref. 29, where Eq. (22) should read

$$
\Lambda_{\omega}=G_{2211}-G_{1111}+\frac{\omega}{2} a_{23111}^{\prime} .
$$

The last equality in the equation $G_{1111}=G_{2211}+G_{1212}$ $+G_{1221}=0$ applies only to the paramagnetic contribution $G_{1111}^{\text {para }}$, whereas for the corresponding diamagnetic term the relationship $G_{1111}^{\mathrm{dia}}=2 G_{2211}^{\mathrm{dia}}+G_{1221}^{\mathrm{dia}} \neq 0$ holds (we have applied straightforwardly the symmetry properties of the diamagnetic susceptibility operator in Eq. (8) above). The contribution of $G_{1111}^{\mathrm{dia}}$ is therefore missing in the data and discussion of Refs. 2, 54, and 60. Note that the following relationships hold, linking the notations used here to those adopted in Ref. 54:

$$
\begin{gathered}
\mathcal{G}_{\text {para }}^{(3)}=-30 G_{1122}^{\text {para }}, \\
\mathcal{G}_{\text {dia }}^{(3)}=30 G_{1111}^{\text {dia }}-30 G_{1122}^{\text {dia }}, \\
\mathcal{A}^{\prime(3)}=-15 \omega a_{23111}^{\prime} .
\end{gathered}
$$

Indeed, it is remarkable that the contribution that was missing in our previous work on atoms is the dominant one in a revised version of Tables II and III of Ref. 54, see the Appendix below. Including it increases the values of $\Lambda_{\omega}$ and, correspondingly, also $\Delta n$ by a considerable factor whose size decreases as the basis set increases. For the largest basis sets in the tables of Ref. 54, this factor varies between 2.5 and 3.5. Coming back to Table II in this paper, this is reflected in the dominant contribution to the Jones constant of the $\mathcal{G}_{\text {dia }}^{(3)}$ term, which always opposes in sign that of $\mathcal{G}_{\text {para }}^{(3)}$ and adds to the definitely minor (albeit significant) contribution of $\mathcal{A}^{\prime(3)}$.

To a first approximation, all the response terms involved in this study should have a power law dependence on the radius of the electronic wave function $r{ }^{88}$ Figure 2 shows the dependencies of the electric dipole polarizability $\alpha$ and the diamagnetic susceptibility $\chi_{m}$ (both experimental results taken from Ref. 89), on the atomic sizes $r_{a}$ (the atomic radii are taken from Ref. 90). In these cases, one would expect to observe a quadratic dependence on $r_{a}$, and, indeed, an anal- ysis of the data yields exponent of 2.3 and 2.1, for $\alpha$ and $\chi_{m}$, respectively, close to what is expected. ${ }^{91}$ Incidentally, fitting calculated values for $\alpha_{\mathrm{av}}$ (obtained as byproducts of our higher order property calculations and reported in Table I) yields an exponent of 2.1. In Figure 2, we also report the product $k_{\mathrm{J}} \lambda$ as a function of $r_{a}$ for the five noble gases studied here, and for the Jones constant we find an exponent of 3.7. This is in rather close agreement with the evidence that the dominant contribution to $k_{\mathrm{J}}$ comes from the $\mathcal{G}_{\text {dia }}^{(3)}$ terms, which should yield in first approximation to an exponent of 4 , whereas all other contributions to $\mathrm{J}_{0}$ would give higher exponents. As a consequence of the trends discussed here for the Jones constant and the electric dipole polarizability, the FOM increases as one moves down the noble gases column of the periodic table, with a nearly quadratic dependence on the atomic radius.

Note that relativistic effects that may become important for heavy atoms like xenon, have been neglected in this study. Plans have been made in our group to further analyse the Jones birefringence of xenon, and in particular relativistic contributions to this effect, in a forthcoming study.

\section{B. Furan homologues and monosubstituted benzenes}

The Jones constant changes sign while moving along the series of furan and its homologues. If the temperatureindependent contribution decreases, while remaining positive, the temperature-dependent term changes sign, its absolute value becoming smaller than the corresponding $\mathrm{J}_{0}$ for thiophene. This behavior is the consequence of the strong changes in the $\mathcal{G}_{\text {para }}^{(2)}$ term, whose absolute value increases by a factor of $\approx 3$ moving down the column from furan, through thiophene to selenophene. The FOM of furan is larger than that of all noble gases, and also of a few of the substituted benzenes, see below. On the other hand, thiophene has the lowest FOM of the whole series in Table I, as low as 0.1. The FOM of selenophene places itself between $\mathrm{Ne}$ and Ar.

The Jones birefringence of benzene was among the properties studied, and in good detail, in Ref. 57. Here, the diamagnetic and paramagnetic $\mathcal{G}_{\text {para }}^{(3)}$ and $\mathcal{G}_{\text {dia }}^{(3)}$ are roughly of the same size, and of opposite sign. They tend to cancel each other and still, at $\lambda=632.8 \mathrm{~nm}$, their sum yields a 

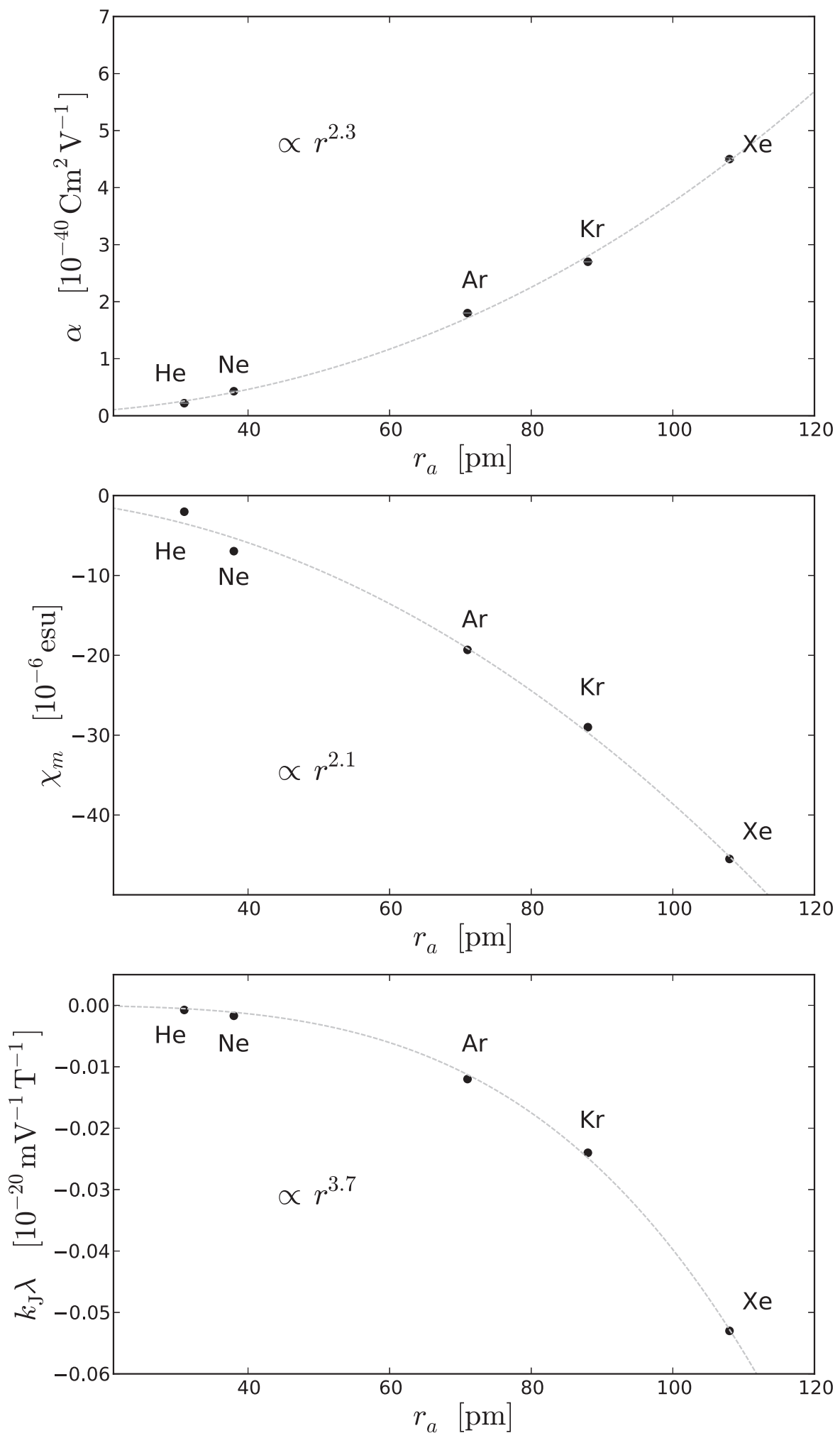

FIG. 2. Dependencies of the experimental values for the polarizability (top), the magnetic susceptibility (middle, absolute values) and the calculated values of $k_{\mathrm{J}} \lambda$ (bottom, again absolute values) on the atomic radius of the noble gases, ranging from He to Xe. Dashed lines are power law fits. For exponents, see text.

contribution which is almost one order of magnitude larger than that of $\mathcal{A}^{\prime(3)}$. Without temperature dependent contributions, the FOM of benzene equals that of $\mathrm{Ne}$.

Among the substituted benzenes, the temperature dependent contribution is smaller (in absolute value) than the temperature independent one for toluene, fluorobenzene, and phenol. In the case of $\mathrm{C}_{6} \mathrm{H}_{5} \mathrm{CH}_{3}$ in particular, the latter is five times larger than the former. In nitrobenzene, the ratio between $T$-dependent and $T$-independent contributions to $k_{\mathrm{J}}$ is $\approx 1.2$. In all other cases, the same ratio assumes values ranging from 3.1 (aminobenzene) to 40.1 (nitrosobenzene). Dominance of the temperature-dependent term implies larger 
Jones constants, and larger FOMs. The latter becomes quite large ( $>20$, for example) for some of the systems in the list, including bromobenzene, benzaldehyde, and cyanobenzene, and surprisingly large for nitrosobenzene (FOM as large as 511).

Going into more details, the sum of $\mathcal{G}_{\text {para }}^{(3)}$ and $\mathcal{G}_{\text {dia }}^{(3)}$ yields a contribution that is between nine and 17 times larger than that of $\mathcal{A}^{\prime(3)}$, and always of opposite sign throughout the list of substituted benzenes. Two exceptions are aminobenzene and nitrosobenzene, where the ratio rises to 33 and 52, respectively. Near cancellations of the $\mathcal{G}_{\text {para }}^{(2)}$ and $\mathcal{G}_{\text {dia }}^{(2)}$ contributions lead to their sum $\mathcal{G}^{(2)}$ being roughly of the same order of magnitude of $\mathcal{A}^{\prime(2)}$ for toluene and fluorobenzene, whereas the $\mathcal{A}^{\prime(2)}$ term is larger than $\mathcal{G}^{(2)}$ for nitrobenzene. In all other cases, this last contribution prevails, being roughly one order of magnitude larger than the $\mathcal{A}^{\prime(2)}$ one, with the resounding exception of nitrosobenzene, where the ratio $\left(\mathcal{G}_{\text {para }}^{(2)}+\mathcal{G}_{\text {dia }}^{(2)}\right) / \mathcal{A}^{\prime(2)}$ is huge, $\approx 1900$.

The series including benzene and 11 of its monosubstituted derivatives is nicely discussed by studying the correlation existing between the Jones constant (actually $k_{\mathrm{J}} \lambda$ ) and the permanent electric dipole or the para Hammett constant. ${ }^{74,75}$ The latter is a qualitative/quantitative descriptor of the relationship between structure and chemical reactivity, a substituent constant which expresses the electron donation and withdrawing capacities of the respective substituent groups, and which has already been seen to correlate nicely with other nonlinear optical properties, e.g., the molecular electric dipole hyperpolarizabilities of substituted benzenes and stilbenes. ${ }^{92}$ Para-Hammett constants for our comparison were taken from Ref. 93. The results of this analysis for the substituted benzenes listed in Table I are shown in Figure 3.

With the relevant exceptions of nitrosobenzene, which clearly sticks out of the list with its huge value and is out of the figure (the Hammett constant of nitrosobenzene being $0.91^{74}$ ), and of nitrobenzene, the Jones constants of all benzenes follow quite nicely a linear relationship, similar to that observed for first electric dipole hyperpolarizabilities. ${ }^{92}$ The correlation between the birefringence and the Hammett constant is clear, including the sign, and it reproduces rather well that observed between birefringence and permanent electric dipole moment. In the figure, we show also the correlation existing between permanent electric dipole and para Hammett constants. Below we will discuss the two relevant exceptions and suggest explications for their "irregular" behavior in Figure 3. As pointed out already in Ref. 92, it is far from trivial to find arguments for the existence of relationships, in particular linear correlations, between an empirical quantity as a substituent constant and a complex nonlinear optical property as the Jones observable. Hammett constants are determined by the influence of a given substituent on the reactivity of a parent compound, and they depend on the ground state electron density at the reaction center. Its relationship with a nonlinear optical property involving the whole electron density, and heavily dependent on the characteristic of the excited state manifold, are rather difficult to ascertain. It is a matter of fact though that a linear relationship is evident in Figure 3. It is also reasonable to state that a correlation para Hammettdipole can be rationalized, since the former describes the elec- tron donating/withdrawing character of the substituent, which also influences the permanent dipole moment of the molecule. Note, from the top panel of Figure 3, how the largest Jones birefringences are associated to large permanent dipole moments, as one would intuitively expect where the $T$-dependent contributions to $k_{\mathrm{J}}$ prevail, due to the role of the electric dipole components in Eqs. (5)-(7).

In order to attempt an explanation for some of the evidences discussed up to this point, it is important to recall that the optical responses involved in the definition of the observable have a complex structure, involving, in their spectral representation, sums over the complete manifold of excited states and the presence of poles, with the insurgence, for particular choices of the excitation frequency, of the phenomenon of resonance enhancement. In comparing frequency dependent responses for a wide selection of systems, it becomes relevant to first identify the positions of the poles in these systems. In the present work, we are adopting standard perturbation theory leading to response functions that become unphysically divergent as optical frequencies approach the values of the transition frequencies, and, as a consequence, the calculated responses can become arbitrarily large in resonance regions. This situation represents an artefact in the formulation of the theory and can be addressed by taking relaxation into account, ${ }^{94}$ which leads to the definition of finite and resonance-convergent response functions.

The vertical electronic excitation energies of the lowlying singlet states for all our studied molecules are summarized in Fig. 4. These are all small aromatic systems with large $\pi \pi *$ transition energies in the region of 4-6 eV. Nitrosobenzene sticks out, however, showing a $n \pi *$-transition at about $1.5 \mathrm{eV}$. An excitation energy as low as $1.5 \mathrm{eV}$ falls below the photon energy associated with our targeted laser operating at $632.8 \mathrm{~nm}$, and, although this state is not very strongly coupled to the electronic ground state by means of the electric-dipole operator, it severely affects the dispersions of the involved response functions and strongly contribute to the Jones effect of this molecule.

In studies of the frequency dependence, it becomes inappropriate to focus on the Jones constant due to the fact that, in comparison to the observable, the wavelength has been factored out [see Eq. (14)]. Instead, it is the frequency dependence of $k_{\mathrm{J}} \lambda$ that is the relevant quantity to study, since it is directly proportional to the birefringence $\Delta n$. In Fig. 5, we present the dispersion of this quantity for nitrosobenzene. In the low frequency limit, the response is about $-10 \times 10^{-20}$ $\mathrm{m}(\mathrm{V} \mathrm{T})^{-1}$ and, at laser detuning of about $0.5 \mathrm{eV}$, the value has roughly doubled and, from there on, the dispersion becomes very strong. Going beyond the resonance energy, there is a sign change in the response property that is associated with sign changes in the denominators of individual response functions for terms involving the first excited state. This explains the fact that, at $632.8 \mathrm{~nm}$, the response property is about $+20 \times 10^{-20} \mathrm{~m}(\mathrm{~V} \mathrm{~T})^{-1}$.

Apart from $\mathrm{C}_{6} \mathrm{H}_{5} \mathrm{NO}$ and for a laser operating at $632.8 \mathrm{~nm}$, the detuning amounts to some $2 \mathrm{eV}$, or more, for all systems in the present study. This is a guarantee for off-resonance conditions and resonance enhancement is therefore expected to be weak. For this reason, it 

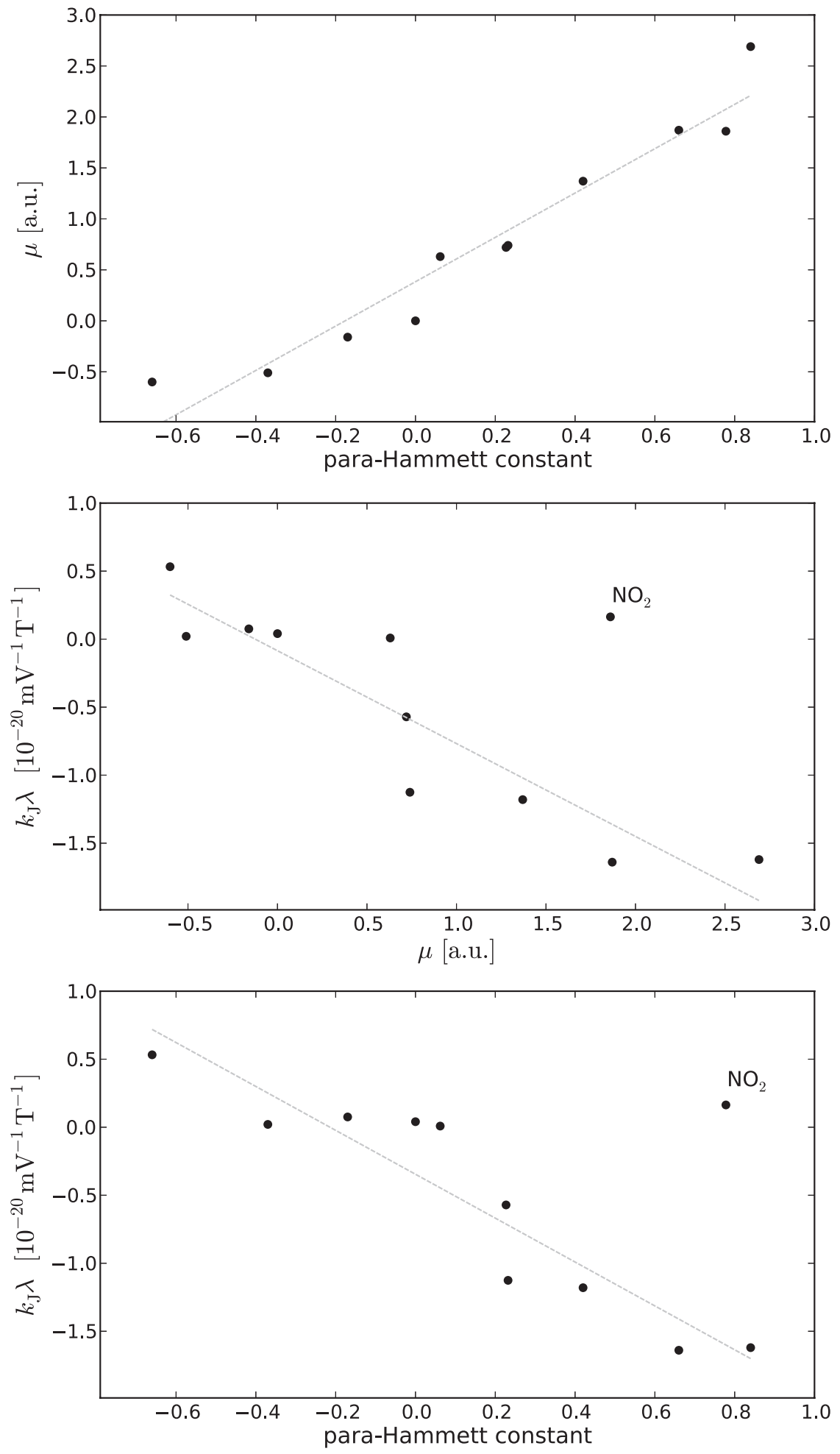

FIG. 3. Calculated Jones constant times the photon wavelength $k_{\mathrm{J}} \lambda$ vs. the corresponding electric dipole permanent moment and the corresponding paraHammett constant for each substituent. Calculations refer to a wavelength of $632.8 \mathrm{~nm}$, pressure of $1 \mathrm{~atm}$, and temperature of $294.15 \mathrm{~K}$, and are carried out at the CAM-B3LYP level of theory. For comparison, the permanent dipole is also reported vs. the corresponding para-Hammett constant.

appears appropriate to compare molecular responses at this common wavelength.

Going back to our correlation between the Jones constant and the para-Hammett constant, we can therefore state that the reason for nitrosobenzene to behave as it does relates to the presence of the $n \pi *$-resonance, but in the case of nitrobenzene the explanation eludes us. For nitrobenzene, we calculate a $\mathrm{J}_{T} / T$ contribution that is relatively small and of the opposite sign as compared to the other nonresonant benzene-acceptor molecules. This explains "numerically" the peculiar behavior of this molecule in Figure 3, see in particular the two low lying panels. Prompted by this evidence, 


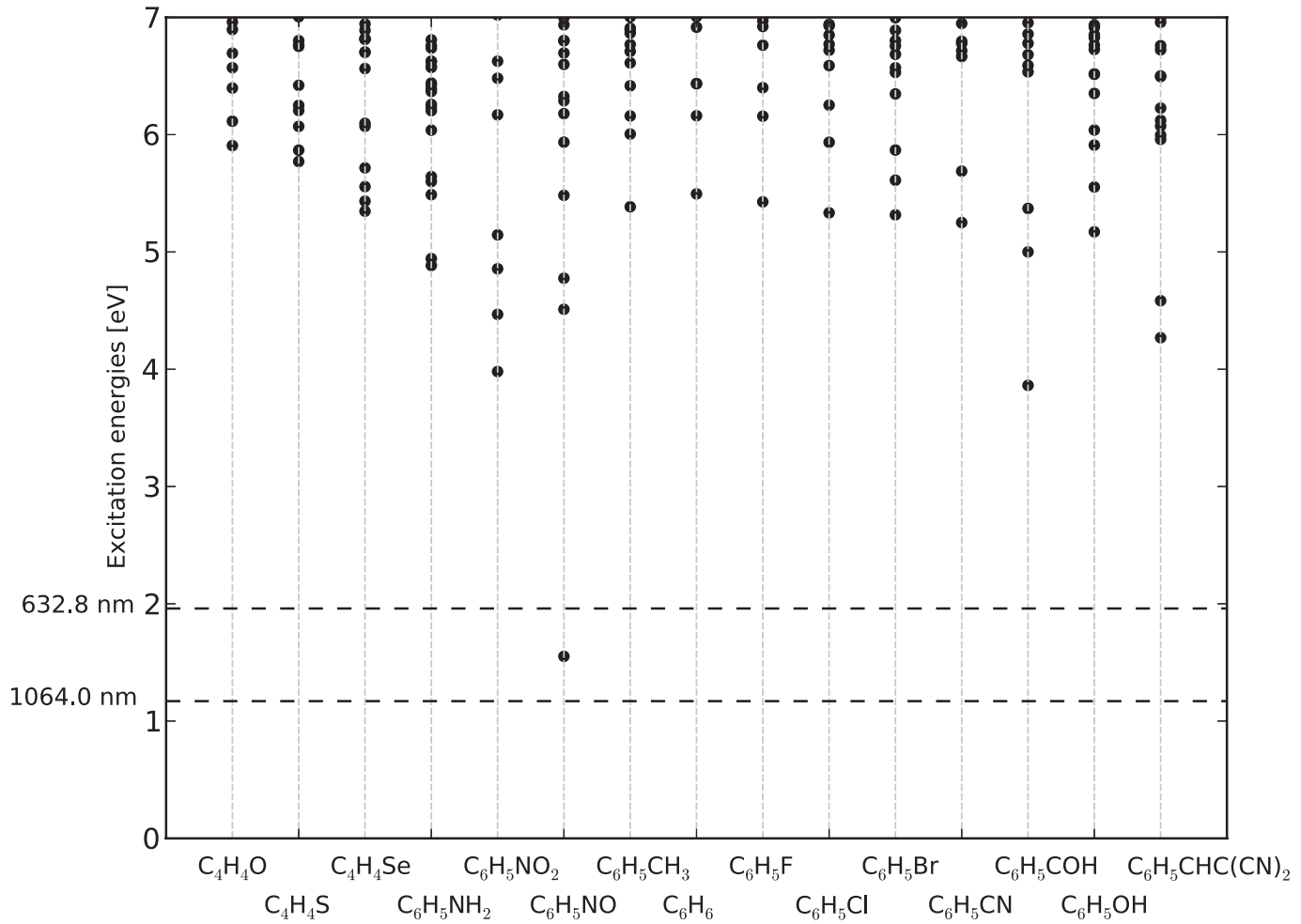

FIG. 4. Vertical electronic excitation energies $(\mathrm{eV})$ for furan homologues and monosubstituted benzenes. The dashed horizontal lines indicate the standard experimental wavelengths of $632.8 \mathrm{~nm}$ and $1064.0 \mathrm{~nm}$.

we have performed additional studies of other properties involving nonlinear frequency dependent responses, for nitrobenzene and for cyanobenzene, selected among the other monosubstituted benzenes studied here. Properties as the first electric dipole hyperpolarizability $\beta(-\omega ; \omega, 0)$ $=-\left\langle\left\langle\hat{\mu}_{\alpha} ; \hat{\mu}_{\beta}, \hat{\mu}_{\gamma}\right\rangle\right\rangle_{\omega, 0}$, or the Verdet constant, measuring the Faraday effect and proportional to the appropriate combina- tion of electric dipole-electric dipole-magnetic dipole first hypersusceptibility tensor components $-\left\langle\left\langle\hat{\mu}_{\alpha} ; \hat{\mu}_{\beta}, \hat{m}_{\gamma}\right\rangle\right\rangle_{\omega, 0}{ }^{3}$ were computed using the same basis set and electronic structure model employed for Jones birefringence. The results well reproduced the expected trends and relationship with experimental data. Apparently the Jones constant is sensitive to a particularity of the nitrobenzene molecule that we have not

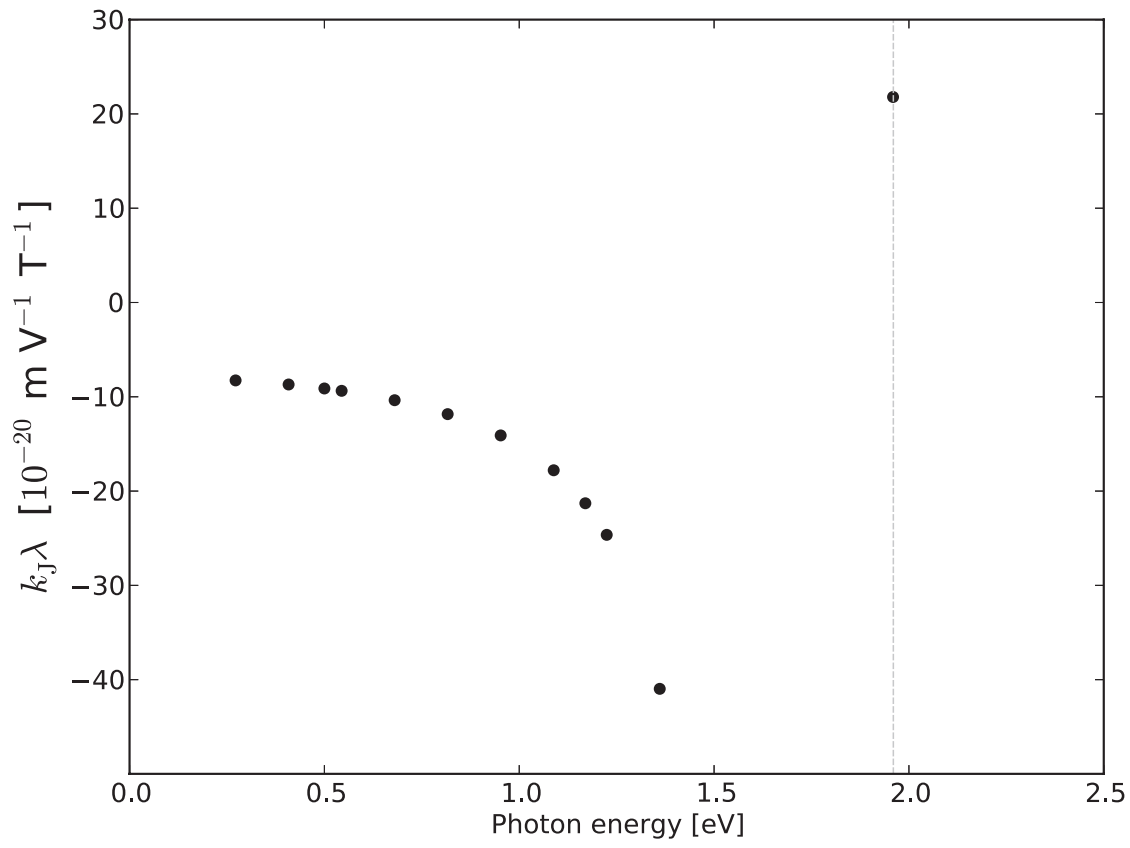

FIG. 5. Dispersion of the Jones constant times the photon wavelength for $\mathrm{C}_{6} \mathrm{H}_{5} \mathrm{NO}$. The dashed vertical line indicates the standard experimental wavelength of $632.8 \mathrm{~nm}(1.96 \mathrm{eV})$. Results refer to a pressure of $1 \mathrm{~atm}$ and a temperature of $294.15 \mathrm{~K}$. 
been able to identify so far, and we cannot offer at this stage physical arguments for the result we obtain in this specific case.

\section{CONCLUSIONS}

An ab initio study of the Jones (and magnetoelectric) birefringence of a series of systems (noble gases, furan and two of its homologues, benzene and 11 monosubstituted derivatives) was carried out employing TDDFT (with CAMB3LYP as the choice of the functional) and large basis sets (Dunning's triply augment correlation consistent of triple- $\zeta$ quality for molecules, of quadruple- $\zeta$ quality for noble gases) with the aim of finding out a system or a class of systems that could be likely candidates for the combined computational/experimental study.

In the course of the analysis, we have come across some interesting and relevant correlations between the observable, the Jones constant, and the atomic radius (for noble gases) or the dipole moment or a substituent empirical parameter as the para Hammett constant, for benzene and its mono substituted derivatives. As an index of the measurability of the birefringence, we have taken a figure of merit defined as the ratio of the product of the Jones constant by the wavelength and the mean static electric dipole polarizability.

The observable follows nicely an expected quadratic law when it comes to the dependence on the atomic radius for noble gases. This includes also Xe, where relativistic effects on the properties have been neglected, prompting us to state that the effect of relativity on the Jones constant of Xe might be negligible.

The linear correlation between Jones constant and para Hammett constant, paralleling that existing between Jones constant and permanent dipole moment, has two notable exceptions: nitrosobenzene and nitrobenzene. Whereas for the former we could identify the reason for the "out of tune" behavior (and for the huge resulting response) in the presence of a nearly resonant excited electronic state, we cannot at present offer a physical argument to explain the behavior of the latter.

\section{ACKNOWLEDGMENTS}

The authors acknowledge support by EuroMagNET II, under EU Contract Number 228043. P.N. acknowledges financial support from the Swedish Research Council (Grant No. 621-2010-5014). S.C. acknowledges support from the Italian Ministero dell'Istruzione, Università e Ricerca (PRIN2009 Grant No. 2009C28YBF_001). The authors acknowledge grants for computing time at National Supercomputer Centre (NSC), Sweden, and at the Italian CINECA.

\section{APPENDIX: REVISION OF DATA FOR NOBLE GASES}

In Tables III and IV we reproduce, for all basis sets employed in Ref. 54 with the exception of the quadruply augmented sets, the relevant tensor elements, and the birefringence computed at full configuration interaction (FCI) level for helium, and CCSD level for neon, argon, and krypton. The notation is that employed in Ref. 54, see also Eqs. (18)-(21) above.

With reference to the discussion of the results obtained for the noble gases in Ref. 54, we note that the $G_{1111}^{\mathrm{dia}}$ contribution, neglected in that paper, is always of opposite sign with respect to and larger (a factor up to more 2.5 in absolute value) than the $G_{2211}^{\mathrm{dia}}$ tensor element, which was seen to be dominant already in Ref. 54. Since the two diamagnetic components contribute with opposite sign to $\Lambda_{\omega}$ (cf. Eq. (20)), the newly introduced all diagonal component yields a sensible increase of $\Lambda_{\omega}$, and a correspondingly enhancement of the birefringence $\Delta n$.

The "best estimates" of the birefringence of the rare gases change accordingly: from $-1.3 \times 10^{-17}$ to $-4.5 \times 10^{-17}$ for helium; from $-3.5 \times 10^{-17}$ to $-1.1 \times 10^{-16}$ for neon; from

TABLE III. He. FCI results for Jones birefringence. Revised version of data of Ref. 54. See text. In parentheses are results from Ref. 54. Wavelength $\lambda=632.8 \mathrm{~nm}$. Birefringences $\Delta n$ computed for a pressure of 1 bar, external electric and magnetic induction fields of $2.6 \times 10^{6} \mathrm{~V} \mathrm{~m}{ }^{-1}$ and $3 \mathrm{~T}$, respectively, and a temperature of $273.15 \mathrm{~K}$.

\begin{tabular}{lcccccc}
\hline \hline Basis & $G_{1111}^{\text {dia }}$ & $G_{2211}^{\text {para }}$ & $G_{2211}^{\text {dia }}$ & $a_{23111}^{\prime}$ & $\Lambda_{\omega}$ & $\Delta n \times 10^{17}$ \\
\hline cc-pVTZ & -0.09675 & -0.04527 & 0.03284 & 0.00252 & $0.08440(-0.01235)$ & $-0.196(0.029)$ \\
cc-pVQZ & -0.27990 & -0.10056 & 0.09288 & 0.01143 & $0.27263(-0.00727)$ & $-0.634(0.017)$ \\
cc-pV5Z & -0.46701 & -0.15175 & 0.16789 & 0.02742 & $0.48414(0.01713)$ & $-1.126(-0.040)$ \\
cc-pV6Z & -0.69374 & -0.20784 & 0.26113 & 0.05434 & $0.74899(0.05525)$ & $-1.742(-0.128)$ \\
aug-cc-pVTZ & -1.45872 & -0.36642 & 0.57747 & 0.19921 & $1.67694(0.21822)$ & $-3.899(-0.507)$ \\
aug-cc-pVQZ & -1.54466 & -0.38672 & 0.71476 & 0.28923 & $1.88311(0.33845)$ & $-4.379(-0.787)$ \\
aug-cc-pV5Z & -1.54804 & -0.39262 & 0.79741 & 0.35784 & $1.96570(0.41767)$ & $-4.571(-0.971)$ \\
aug-cc-pV6Z & -1.49822 & -0.39322 & 0.85004 & 0.41112 & $1.96983(0.47162)$ & $-4.580(-1.097)$ \\
d-aug-cc-pVTZ & -1.37139 & -0.39407 & 0.91118 & 0.53083 & $1.90760(0.53622)$ & $-4.436(-1.247)$ \\
d-aug-cc-pVQZ & -1.38055 & -0.39524 & 0.92584 & 0.53388 & $1.93036(0.54982)$ & $-4.489(-1.278)$ \\
d-aug-cc-pV5Z & -1.39391 & -0.39456 & 0.92935 & 0.53548 & $1.94797(0.55407)$ & $-4.530(-1.288)$ \\
d-aug-cc-pV6Z & -1.40124 & -0.39400 & 0.92662 & 0.53492 & $1.95312(0.55188)$ & $-4.542(-1.283)$ \\
t-aug-cc-pVTZ & -1.37416 & -0.39457 & 0.91193 & 0.53323 & $1.91072(0.53655)$ & $-4.443(-1.248)$ \\
t-aug-cc-pVQZ & -1.37990 & -0.39528 & 0.92569 & 0.53433 & $1.92955(0.54964)$ & $-4.487(-1.278)$ \\
t-aug-cc-pV5Z & -1.39133 & -0.39446 & 0.92914 & 0.53491 & $1.94526(0.55394)$ & $-4.523(-1.288)$ \\
t-aug-cc-pV6Z & -1.39928 & -0.39392 & 0.92679 & 0.53456 & $1.95139(0.55211)$ & $-4.538(-1.284)$ \\
\hline \hline
\end{tabular}


TABLE IV. CCSD results for Jones birefringence of Neon, Argon, and Krypton. Revised version of data of Ref. 54. See text. In parentheses are results from Ref. 54. Wavelength $\lambda=632.8 \mathrm{~nm}$. Birefringences $\Delta n$ computed for a pressure of $1 \mathrm{bar}$, external electric and magnetic induction fields of $2.6 \times 10^{6} \mathrm{~V} \mathrm{~m}^{-1}$ and $3 \mathrm{~T}$, respectively, and a temperature of $273.15 \mathrm{~K}$.

\begin{tabular}{|c|c|c|c|c|c|c|}
\hline Basis & $G_{1111}^{\mathrm{dia}}$ & $G_{2211}^{\text {para }}$ & $G_{2211}^{\mathrm{dia}}$ & $a_{23111}^{\prime}$ & $\Lambda_{\omega}$ & $\Delta n \times 10^{17}$ \\
\hline \multicolumn{7}{|c|}{ Neon } \\
\hline aug-cc-pVTZ & -2.22662 & -0.55093 & 1.02835 & 0.83594 & $2.73414(0.50752)$ & $-6.358(-1.180)$ \\
\hline aug-cc-pVQZ & -2.69983 & -0.63667 & 1.37156 & 1.00038 & $3.47074(0.77091)$ & $-8.070(-1.793)$ \\
\hline aug-cc-pV5Z & -2.96323 & -0.68655 & 1.70140 & 1.19172 & $4.02098(1.05775)$ & $-9.350(-2.460)$ \\
\hline d-aug-cc-pVTZ & -3.50756 & -0.75070 & 1.85796 & 1.41982 & $4.66594(1.15838)$ & $-10.850(-2.694)$ \\
\hline d-aug-cc-pVQZ & -3.25614 & -0.73992 & 2.00568 & 1.50209 & 4.57597 (1.31983) & $-10.640(-3.069)$ \\
\hline d-aug-cc-pV5Z & -3.06233 & -0.73404 & 2.15598 & 1.63683 & $4.54320(1.48087)$ & $-10.564(-3.443)$ \\
\hline t-aug-cc-pVTZ & -3.19133 & -0.75633 & 2.17231 & 1.67606 & 4.66765 (1.47632) & $-10.854(-3.433)$ \\
\hline t-aug-cc-pVQZ & -3.04631 & -0.74037 & 2.17100 & 1.68486 & $4.53759(1.49128)$ & $-10.551(-3.468)$ \\
\hline t-aug-cc-pV5Z & -3.02514 & -0.73399 & 2.18048 & 1.69322 & $4.53258(1.50744)$ & $-10.540(-3.505)$ \\
\hline \multicolumn{7}{|c|}{ Argon } \\
\hline aug-cc-pVTZ & -21.72543 & -6.46468 & 12.32241 & 18.42279 & $28.24641(6.52098)$ & $-65.681(-15.163)$ \\
\hline aug-cc-pVQZ & -24.97387 & -7.08445 & 15.77958 & 23.09026 & $34.50028(9.52641)$ & $-80.223(-22.152)$ \\
\hline aug-cc-pV5Z & -22.52025 & -7.01763 & 16.99101 & 24.62570 & $33.38019(10.85994)$ & $-77.618(-25.252)$ \\
\hline d-aug-cc-pVTZ & -25.14808 & -7.45078 & 18.81484 & 29.54271 & $37.57572(12.42764)$ & $-87.374(-28.898)$ \\
\hline d-aug-cc-pVQZ & -23.66531 & -7.30320 & 19.13405 & 29.77622 & $36.56814(12.90283)$ & $-85.031(-30.003)$ \\
\hline d-aug-cc-pV5Z & -22.58023 & -7.25819 & 19.58557 & 31.11452 & $36.02777(13.44754)$ & $-83.775(-31.269)$ \\
\hline t-aug-cc-pVTZ & -23.28978 & -7.45600 & 20.30386 & 33.65619 & $37.34931(14.05953)$ & $-86.848(-32.692)$ \\
\hline t-aug-cc-pVQZ & -22.60071 & -7.30326 & 19.93156 & 32.26406 & $36.39056(13.78985)$ & $-84.618(-32.065)$ \\
\hline t-aug-cc-pV5Z & -22.35573 & -7.25834 & 19.73956 & 31.90732 & $35.98566(13.62993)$ & $-83.677(-31.693)$ \\
\hline \multicolumn{7}{|c|}{ Krypton } \\
\hline aug-cc-pVTZ & -49.24551 & -14.22440 & 28.15406 & 56.19013 & $65.19809(15.95258)$ & $-151.604(-37.094)$ \\
\hline aug-cc-pVQZ & -48.32808 & -14.54613 & 33.68654 & 64.39986 & $69.78697(21.45889)$ & $-162.274(-49.898)$ \\
\hline aug-cc-pV5Z & -44.89622 & -14.41114 & 36.13720 & 69.18110 & $69.11290(24.21668)$ & $-160.707(-56.311)$ \\
\hline d-aug-cc-pVTZ & -51.16270 & -15.60627 & 39.73010 & 82.25847 & $78.24796(27.08526)$ & $-181.948(-62.981)$ \\
\hline d-aug-cc-pVQZ & -46.69773 & -15.11992 & 40.90421 & 84.20056 & $75.51336(28.81563)$ & $-175.590(-67.004)$ \\
\hline d-aug-cc-pV5Z & -44.52121 & -14.83324 & 40.96292 & 84.65747 & $73.69867(29.17747)$ & $-171.370(-67.846)$ \\
\hline t-aug-cc-pVTZ & -46.80882 & -15.60989 & 43.02425 & 93.66344 & $77.59519(30.78637)$ & $-180.431(-71.587)$ \\
\hline t-aug-cc-pVQZ & -44.97673 & -15.11689 & 42.17999 & 90.08271 & $75.28293(30.30620)$ & $-175.054(-70.470)$ \\
\hline t-aug-cc-pV5Z & -43.96590 & -14.83800 & 41.44806 & 87.58322 & $73.72908(29.76318)$ & $-171.441(-69.208)$ \\
\hline
\end{tabular}

$-3.0 \times 10^{-16}$ to $-8.4 \times 10^{-16}$ for argon; and from -6.5 $\times 10^{-16}$ to $-1.7 \times 10^{-15}$ for krypton.

${ }^{1}$ L. D. Barron, Molecular Light Scattering and Optical Activity (Cambridge University Press, Cambridge, 2004).

${ }^{2}$ A. Rizzo and S. Coriani, Adv. Quantum Chem. 50, 143 (2005).

${ }^{3}$ M. Jaszunski, A. Rizzo, and K. Ruud, "Molecular electric, magnetic and optical properties," in Handbook of Computational Chemistry, edited by J. Leszczynski (Springer Science (in two volumes) + Business Media (in three volumes), 2012). Vol. 1, Chap. 11, pp. 361-441.

${ }^{4}$ D. J. Caldwell and H. Eyring, The Theory of Optical Activity (Wiley Interscience, New York, 1971).

${ }^{5}$ J. A. Schellman, Chem. Rev. 75, 323 (1975).

${ }^{6}$ Circular Dichroism: Principles and Applications, 2nd ed., edited by N. Berova, K. Nakanishi, and R. W. Woody (Wiley, New York, 2000).

${ }^{7}$ Comprehensive Chiroptical Spectroscopy, edited by N. Berova, P. L. Polavarapu, K. Nakanishi, and R. W. Woody (Wiley, New York, 2012).

${ }^{8}$ J. Kerr, Philos. Mag. 50, 337 (1875).

${ }^{9}$ J. Kerr, Philos. Mag. 50, 446 (1875).

${ }^{10}$ A. D. Buckingham, Proc. Phys. Soc. B 69, 344 (1956).

${ }^{11}$ G. Mayer and F. Gires, C. R. Hebd. Séances Acad. Sci., Paris 258, 2039 (1964).

${ }^{12}$ P. P. Ho and R. R. Alfano, Phys. Rev. A 20, 2170 (1979).

${ }^{13}$ Q. Majorana, Rend. Accad. Naz. Lincei 11, 374 (1902).

${ }^{14}$ Q. Majorana, C. R. Hebd. Séances Acad. Sci., Paris 135, 159 (1902).

${ }^{15}$ Q. Majorana, C. R. Hebd. Séances Acad. Sci., Paris 135, 235 (1902).
${ }^{16}$ A. Cotton and M. Mouton, C. R. Hebd. Séances Acad. Sci., Paris 141, 317 (1905).

${ }^{17}$ A. Cotton and M. Mouton, C. R. Hebd. Séances Acad. Sci., Paris 141, 349 (1905).

${ }^{18}$ A. Cotton and M. Mouton, C. R. Hebd. Séances Acad. Sci., Paris 145, 229 (1907).

${ }^{19}$ A. Cotton and M. Mouton, C. R. Hebd. Séances Acad. Sci., Paris 145, 870 (1907).

${ }^{20}$ A. D. Buckingham and J. A. Pople, Proc. Phys. Soc. B 69, 1133 (1956).

${ }^{21}$ A. D. Buckingham, J. Chem. Phys. 30, 1580 (1959).

${ }^{22}$ A. D. Buckingham and R. L. Disch, Proc. Roy. Soc. A 273, 275 (1963).

${ }^{23}$ A. D. Buckingham and H. C. Longuet-Higgins, Mol. Phys. 14, 63 (1968).

${ }^{24}$ A. D. Buckingham and M. J. Jamieson, Mol. Phys. 22, 117 (1971).

${ }^{25}$ D. A. Imrie and R. E. Raab, Mol. Phys. 74, 833 (1991).

${ }^{26}$ R. E. Raab and O. L. de Lange, Mol. Phys. 101, 3467 (2003).

${ }^{27}$ O. L. de Lange and R. E. Raab, Mol. Phys. 102, 125 (2004).

${ }^{28}$ R. C. Jones, J. Opt. Soc. Am. 38, 671 (1948).

${ }^{29}$ E. B. Graham and R. E. Raab, Proc. R. Soc. London, Ser. A 390, 73 (1983).

${ }^{30}$ F. Pockels, Radium 10, 152 (1913).

${ }^{31}$ E. B. Graham and R. E. Raab, Mol. Phys. 52, 1241 (1984).

${ }^{32} \mathrm{~S}$. Kielich, in Molecular Electro-Optics, edited by C. T. O'Konski (Marcel Dekker, New York, 1976).

${ }^{33}$ N. B. Baranova, Y. V. Bogdanov, and B. Y. Zel'dovich, Sov. Phys. Usp. 20, 870 (1977).

${ }^{34}$ H. J. Ross, B. S. Sherborne, and G. E. Stedman, J. Phys. B 22, 459 (1989).

${ }^{35}$ M. Faraday, Philos. Mag. 28, 294 (1846).

${ }^{36}$ M. Faraday, Philos. Trans. R. Soc. 136, 1 (1846). 
${ }^{37}$ J. Michal and E. W. Thulstrup, Spectroscopy with Polarized Light $(\mathrm{VCH}$ Publishers, Inc., New York, 1986).

${ }^{38}$ P. W. Atkins and M. H. Miller, Mol. Phys. 15, 503 (1968).

${ }^{39}$ L. D. Barron and J. Vrbancich, Mol. Phys. 51, 715 (1984).

${ }^{40}$ N. G. Kalugin, P. Kleindienst, and G. H. Wagniére, Chem. Phys. 248, 105 (1999).

${ }^{41}$ P. J. Stephens, J. Chem. Phys. 52, 3489 (1970).

${ }^{42}$ E. W. Thulstrup, Aspects of the Linear Magnetic Circular Dichroism of Planar Organic Molecules (Springer-Verlag, Berlin, 1980).

${ }^{43}$ W. R. Mason, A Practical Guide to Magnetic Circular Dichroism Spectroscopy (Wiley, New York, 2007).

${ }^{44}$ S. B. Piepho and P. N. Schatz, Group Theory in Spectroscopy: With Applications to Magnetic Circular Dichroism (Wiley, New York, 1983).

${ }^{45}$ A. D. Buckingham and P. J. Stephens, Annu. Rev. Phys. Chem. 17, 399 (1966).

${ }^{46}$ P. N. Schatz and A. J. McCaffery, Q. Rev. 23, 552 (1969).

${ }^{47}$ P. J. Stephens, Annu. Rev. Phys. Chem. 25, 201 (1974).

${ }^{48}$ P. J. Stephens, Adv. Chem. Phys. 35, 197 (1976).

${ }^{49}$ P. J. Stephens, Chem. Phys. Lett. 2, 241 (1968).

${ }^{50}$ G. L. J. A. Rikken and E. Raupach, Nature (London) 390, 493 (1997).

${ }^{51}$ G. L. J. A. Rikken and E. Raupach, Nature (London) 405, 932 (2000).

${ }^{52}$ Y. Kitagawa, H. Segawa, and K. Ishii, Angew. Chem., Int. Ed. 50, 9133 (2011).

${ }^{53}$ Y. Kitagawa, T. Miyatake, and K. Ishii, Chem. Commun. 48, 5091 (2012).

${ }^{54}$ A. Rizzo and S. Coriani, J. Chem. Phys. 119, 11064 (2003).

${ }^{55}$ E. Runge and E. K. U. Gross, Phys. Rev. Lett. 52, 997 (1984).

${ }^{56}$ M. A. L. Marques and E. K. U. Gross, Annu. Rev. Phys. Chem. 55, 427 (2004).

${ }^{57}$ A. Rizzo, C. Cappelli, B. Jansík, D. Jonsson, P. Sałek, S. Coriani, and H. Ågren, J. Chem. Phys. 121, 8814 (2004); 129, 039901 (2008) (Erratum).

${ }^{58}$ A. Rizzo, C. Cappelli, B. Jansík, D. Jonsson, P. Sałek, S. Coriani, D. J. D. Wilson, T. U. Helgaker, and H. Ågren, J. Chem. Phys. 122, 234314 (2005); 129, 039901 (2008) (Erratum).

${ }^{59}$ G. D. Purvis and R. J. Bartlett, J. Chem. Phys. 76, 1910 (1982).

${ }^{60} \mathrm{O}$. Christiansen, S. Coriani, J. Gauss, C. Hättig, P. Jørgensen, F. Pawłowski, and A. Rizzo, "Accurate NLO properties for small molecules: Methods and results," in Non-Linear Optical Properties of Matter: From Molecules to Condensed Phases, Challenges and Advances in Computational Chemistry and Physics Vol. 1, edited by M. G. Papadopoulos, A. J. Sadlej, and J. Leszczynski (Springer, Dordrecht, The Netherlands, 2006), pp. 51-99.

${ }^{61}$ A. Rizzo and C. Cappelli, Int. J. Quantum Chem. 111, 760 (2011).

${ }^{62}$ A. Rizzo, D. Shcherbin, and K. Ruud, Can. J. Chem. 87, 1352 (2009).

${ }^{63}$ D. Shcherbin, A. J. Thorvaldsen, D. Jonsson, and K. Ruud, J. Chem. Phys. 135, 134114 (2011).

${ }^{64}$ P. V. Mironova, V. D. Ovsiannikov, and V. V. Chernushkin, J. Phys. B 39, 4999 (2006).

${ }^{65}$ O. Arteaga, Opt. Lett. 35, 1359 (2010).

${ }^{66}$ T. Roth and G. L. J. A. Rikken, Phys. Rev. Lett. 85, 4478 (2000).

${ }^{67} \mathrm{~T}$. Roth, "Experimental verification of the Jones birefringence induced in liquids," Diplomarbeit, Darmstadt University of Technology and Grenoble High Magnetic Field Laboratory, 2000.

${ }^{68}$ T. Roth and G. L. J. A. Rikken, "Magneto-electric Jones birefringence: A bianisotropic effect," in Proceedings of the 8th International Conference on Electromagnetics of Complex Media, Lisbon, Portugal, 27-29 September 2000, Bianisotropics 2000, Technical Report No. ADPO 11633 (Defense Technical Information Center, 2000).

${ }^{69}$ G. L. J. A. Rikken, E. Raupach, and T. Roth, Physica B 294-295, 1 (2001).
${ }^{70}$ T. Roth and G. L. J. A. Rikken, Phys. Rev. Lett. 88, 063001 (2002).

${ }^{71}$ P. Sałek, O. Vahtras, T. Helgaker, and H. Ågren, J. Chem. Phys. 117, 9630 (2002).

${ }^{72}$ B. Jansík, P. Sałek, D. Jonsson, O. Vahtras, and H. Ågren, J. Chem. Phys. 122, 054107 (2005)

${ }^{73}$ A. Rizzo, S. Coriani, and K. Ruud, "Response function theory computational approaches to linear and non-linear optical spectroscopy," in Computational Strategies for Spectroscopy: From Small Molecules to Nano Systems, edited by V. Barone (John Wiley \& Sons, Hoboken, NJ, 2012). Chap. 2, pp. 77-135.

${ }^{74}$ C. Hansch, A. Leo, and R. W. Taft, Chem. Rev. 91, 165 (1991).

${ }^{75} \mathrm{~J}$. March, Advanced Organic Chemistry: Reactions, Mechanisms and Structure (Wiley \& Sons, New York, 1998).

${ }^{76}$ A. D. Becke, J. Chem. Phys. 98, 5648 (1993).

${ }^{77}$ A. D. Becke, Phys. Rev. A 38, 3098 (1988).

${ }^{78}$ C. Lee, W. Yang, and R. G. Parr, Phys. Rev. B 37, 785 (1988).

${ }^{79}$ T. H. Dunning, J. Chem. Phys. 90, 1007 (1989).

${ }^{80}$ Y. Yanai, D. P. Tew, and N. C. Handy, Chem. Phys. Lett. 393, 51 (2004).

${ }^{81}$ M. J. G. Peach, T. Helgaker, P. Sałek, T. W. Keal, O. B. Lutnæs, D. J. Tozer, and N. C. Handy, Phys. Chem. Chem. Phys. 8, 558 (2006).

${ }^{82}$ M. J. Paterson, O. Christiansen, F. Pawłowski, P. Jørgensen, C. Hättig, T. Helgaker, and P. Sałek, J. Chem. Phys. 124, 054322 (2006)

${ }^{83}$ D. E. Woon and T. H. Dunning, Jr., J. Chem. Phys. 98, 1358 (1993).

${ }^{84}$ D. E. Woon and T. H. Dunning, Jr., J. Chem. Phys. 100, 2975 (1994).

${ }^{85}$ K. Peterson, D. Figgen, E. Goll, H. Stoll, and M. Dolg, J. Chem. Phys. 119, 11113 (2003).

${ }^{86}$ M. J. Frisch, G. W. Trucks, H. B. Schlegel et al., Gaussian 03, Revision B05, Gaussian, Inc., Pittsburgh, PA, 2003.

${ }^{87}$ K. Aidas, C. Angeli, K. L. Bak, V. Bakken, R. Bast, L. Boman, O. Christiansen, R. Cimiraglia, S. Coriani, P. Dahle, E. K. Dalskov, U. Ekström, T. Enevoldsen, J. J. Eriksen, P. Ettenhuber, B. Fernández, L. Ferrighi, H. Fliegl, L. Frediani, K. Hald, A. Halkier, C. Hättig, H. Heiberg, T. Helgaker, A. C. Hennum, H. Hettema, E. Hjertenæs, S. Høst, I.-M. Høyvik, M. F. Iozzi, B. Jansik, H. J. Aa. Jensen, D. Jonsson, P. Jørgensen, J. Kauczor, S. Kirpekar, T. Kjærgaard, W. Klopper, S. Knecht, R. Kobayashi, H. Koch, J. Kongsted, A. Krapp, K. Kristensen, A. Ligabue, O. B. Lutnæs, J. I. Melo, K. V. Mikkelsen, R. H. Myhre, C. Neiss, C. B. Nielsen, P. Norman, J. Olsen, J. M. H. Olsen, A. Osted, M. J. Packer, F. Pawlowski, T. B. Pedersen, P. F. Provasi, S. Reine, Z. Rinkevicius, T. A. Ruden, K. Ruud, V. Rybkin, P. Salek, C. C. M. Samson, A. Sánchez de Merás, T. Saue, S. P. A. Sauer, B. Schimmelpfennig, K. Sneskov, A. H. Steindal, K. O. SylvesterHvid, P. R. Taylor, A. M. Teale, E. I. Tellgren, D. P. Tew, A. J. Thorvaldsen, L. Thøgersen, O. Vahtras, M. Watson, D. J. Wilson, M. Ziolkowski, and H. Ågren, "The Dalton quantum chemistry program system," WIREs Comput. Mol. Sci. (published online).

${ }^{88}$ P. W. Atkins and R. Friedman, Molecular Quantum Mechanics, 4th ed. (Oxford University Press, Oxford, UK, 2005), Chap. 12.

${ }^{89}$ CRC Handbook of Chemistry and Physics, edited D. R. Lide (CRC Press, Boca Raton, FL, 2005) (Internet version, see http://www.hbcpnetbase.com).

${ }^{90}$ E. Clementi, D. L. Raimondi, and W. P. Reinhardt, J. Chem. Phys. 47, 1300 (1967).

${ }^{91}$ P. Politzer, P. Jin, and J. S. Murray, J. Chem. Phys. 117, 8197 (2002).

${ }^{92}$ L.-T. Cheng, W. Tam, S. H. Stevenson, G. R. Meredith, G. Rikken, and S. R. Marder, J. Phys. Chem. 95, 10631 (1991).

${ }^{93}$ J. E. Leffler and E. Grunwald, Rates and Equilibria of Organic Reactions (Dover, Mineola, 1989).

${ }^{94}$ P. Norman, Phys. Chem. Chem. Phys. 13, 20519 (2011). 